\title{
Simultaneous transarterial and transvenous contrast injection to reveal the fistulous point in carotid cavernous fistula: illustrative case
}

\author{
Chintan Rupareliya, MD, MHA, ${ }^{1}$ Justin F. Fraser, MD, ${ }^{1-5}$ and Lila Sheikhi, $\mathrm{MD}^{1-3}$ \\ Departments of ${ }^{1}$ Neurology, ${ }^{2}$ Neurosurgery, ${ }^{3}$ Radiology, ${ }^{4}$ Neuroscience, and ${ }^{5}$ Center for Advanced Translational Stroke Science, University of Kentucky, Lexington, Kentucky
}

BACKGROUND Access of the cavernous sinus (CS) via venous route from the inferior petrosal sinus (IPS) can sometimes be challenging during the treatment of carotid cavernous fistulas (CCF), largely because of anatomical variations, tortuosity, and/or difficult visualization of IPS given high retrograde flow through the fistulous connection.

OBSERVATIONS A 58-year-old male was transferred to our university hospital center after suspected diagnosis of CCF at another hospital by head computerized tomography-angiogram. His symptoms included three weeks of right eye pain that was later complicated by redness, diplopia, and blurry vision. In a diagnostic angiogram, separate contrast injections from the arterial side via internal carotid artery (ICA) and from the venous side via IPS did not reveal a connection point. Injecting contrast simultaneously from both arterial and venous ends resulted in visualization of a connection point allowing entry into the CS.

LESSONS Technique of simultaneous contrast injection from ICA and internal jugular vein is comparatively simple and saves an operator prolonged time and complexity of approach. In our case, it revealed fistulous point allowing navigation and completing the coiling.

https://thejns.org/doi/abs/10.3171/CASE21456

KEYWORDS carotid cavernous fistula; simultaneous contrast injection; CCF; CCF embolization

Endovascular approach remains the gold standard approach to treat carotid cavernous fistulas (CCFs). Accessing the cavernous sinus (CS) via the venous route from the inferior petrosal sinus (IPS) is the favored approach given the shortest course of IPS via low pressure internal jugular vein (IJV). ${ }^{1}$ A major hurdle in accessing the IPS is the fast transit of contrast via fistulous point secondary to high pressure from arterial end. Various techniques of accessing the IPS have been reported in the literature.

The aim of this article is to report a technique of simultaneously injecting contrast from arterial system via the internal carotid artery (ICA) and venous system via IPS opacifying the connection point and allowing navigation into the IPS.

\section{Illustrative Case}

A 58-year-old male was transferred to our university hospital center after suspected diagnosis of CCF at an outside hospital by head computerized tomography-angiogram (CTA). His symptoms included three weeks of right eye pain that was later complicated by redness, diplopia, and blurry vision. Brain magnetic resonance imaging (MRI) showed hemorrhagic lesion in the right parietotemporal region and CTA demonstrated CS filling in early arterial phase. Due to limited sequencing of the outside MRI, a repeat MRI brain was obtained at our center that confirmed hemorrhagic lesion in the right temporal region measuring $20 \times 12 \times 5 \mathrm{~mm}$. After reviewing imaging, a decision was made to perform digital subtraction angiogram (DSA) that confirmed the CCF. Given the presence of symptoms and hematoma, embolization using an endovascular approach was planned.

General anesthesia was used. The arterial system was accessed from right common femoral artery. A 4F Cook catheter (Cook Medical LLC) was advanced over 0.035 angled glide wire to the proximal right ICA. Contrast injected though the ICA showed the CS but not the IPS (Fig. 1A). The venous system was accessed via left

\footnotetext{
ABBREVIATIONS CCA = common carotid artery; CCF = carotid cavernous fistula; $\mathrm{CS}=$ cavernous sinus; CTA = computerized tomography-angiogram; DSA = digital subtraction angiogram; ICA = internal carotid artery; IJV = internal jugular vein; IPS = inferior petrosal sinus; IVUS = intravascular ultrasound; MRI = magnetic resonance imaging; PCom $=$ posterior communicating.

INCLUDE WHEN CITING Published February 21, 2022; DOI: 10.3171/CASE21456.
}

SUBMITTED August 18, 2021. ACCEPTED January 7, 2022.

(C) 2022 The authors, CC BY-NC-ND 4.0 (http://creativecommons.org/licenses/by-nc-nd/4.0/). 

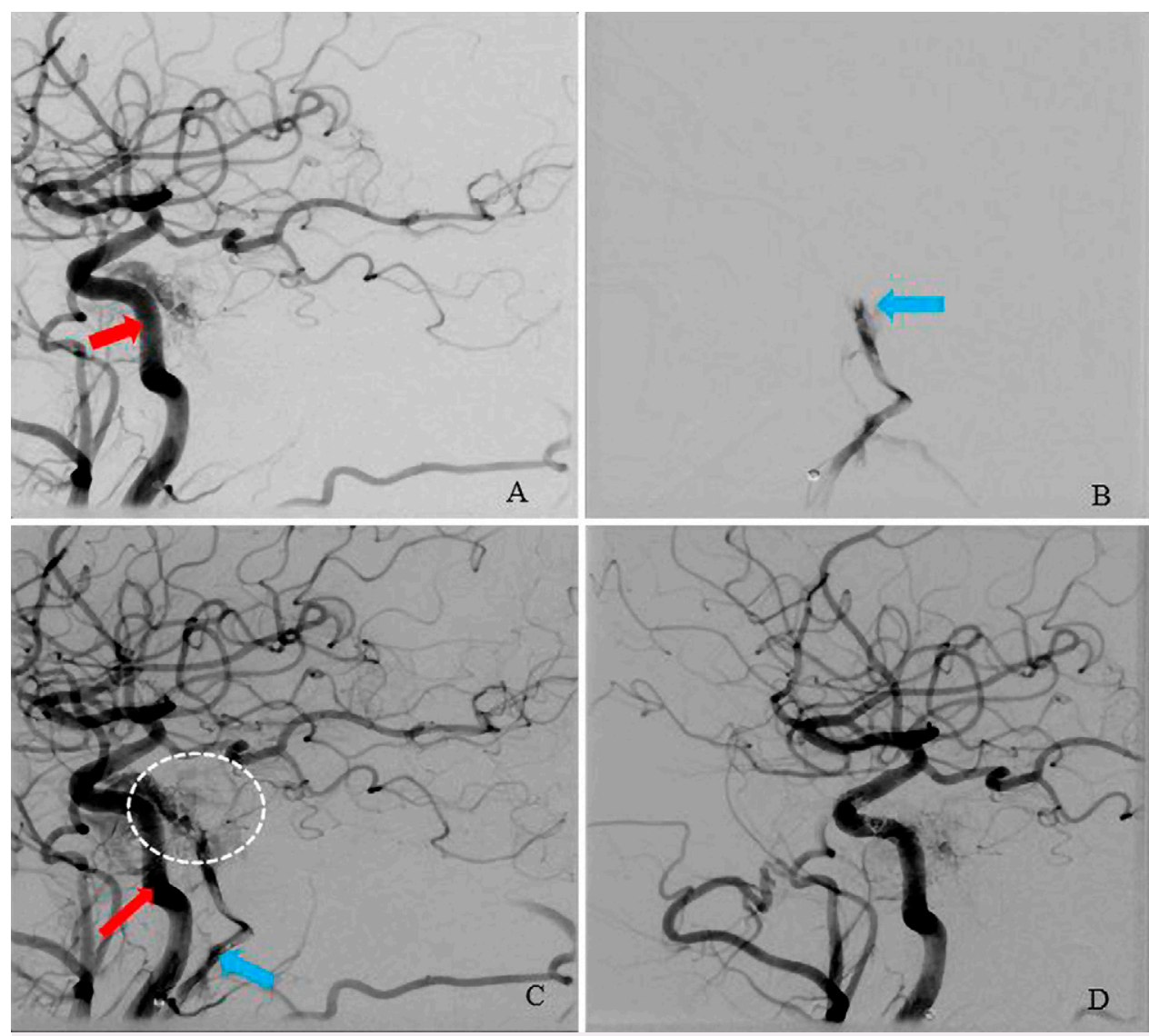

FIG. 1. A: Injection into the right ICA (red arrow) opacifies cavernous sinus, but no flow into IPS. B: Injection into the right IJV (blue arrow) opacifies half of IPS, but not the connection point or cavernous sinus. C: Simultaneous contrast injection into the right ICA (red arrow) and right IJV (blue arrow) revealing connection point (white dashed circle) allowing subsequent navigation. D: Right ICA injection after successful coil embolization showing resolution.

common femoral vein, using an Infinity guide catheter (Stryker Neurovascular) and Catalyst 5 (Stryker Neurovascular) distal access catheter. Through Echelon 10 (Medtronic) microcatheter, Synchro 2 soft microwire (Stryker Neurovascular) was advanced and venous guide catheter was placed into right IJV. After placing distal access catheter into sigmoid jugular junction, contrast was injected that revealed only a part of IPS and not the CS (Fig. 1B). Later, contrast was injected with the microcatheter within the proximal IPS and the diagnostic catheter in the left ICA elucidated the CS-IPS connection (Figs. $1 \mathrm{C}$ and 2) allowing for subsequent navigation and complete treatment using target coils (Fig. 1D).

\section{Discussion}

CCF is a pathological connection point between the high-pressure carotid artery and low-pressure CS. ${ }^{1}$ CCFs are classified into traumatic versus spontaneous based on etiology, high flow versus low flow based on hemodynamics, and direct versus indirect based on the angioarchitecture. ${ }^{1,2}$ Untreated symptomatic CCFs may cause bidirectional damage: long-term fistula results in an engorgement of ipsilateral ocular veins resulting in retinal damage as a pressure effect and compromise in the antegrade flow into the ipsilateral carotid system.
Surgical treatment of CCFs has been used since the early 1930s comprising ligation of the ICA. Packing of the CS after surgical exposure was performed by Parkinson et al. ${ }^{1}$ The endovascular era of CCF management began in 1974 with Serbinenko successfully embolizing the CS with a detachable balloon. ${ }^{1}$ This technique remained popular until 2003 when the devices were withdrawn from the U.S. market because of problems with detachments. As a result, the use of an alternative embolizing material (e.g., platinum coils; Trufill, Onyx, etc.) increased and currently are a mainstay of treatment. ${ }^{1}$

The transvenous approach (also known as the posterior approach) is commonly used for endovascular treatment of indirect CCF. ${ }^{1,4}$ Success of transvenous approach mainly relies on patency of IPS and degree of tortuosity between IJV and IPS. ${ }^{1}$ Challenges encountered while pursuing transvenous route may include but are not limited to: anatomical variations, acute angle between IJV and IPS, narrow lumen of IJV, clotting of IJV, high backflow pressure from ICA, fast transit of contrast through fistulous connection offering less time to interpret the anatomy, and flow directionality for further intervention. ${ }^{1,3}$

Many techniques have been described in the literature to access a difficult IPS. In 1975, Huber ${ }^{5}$ used a technique of vertebral artery contrast injection with simultaneous digital compression of the 


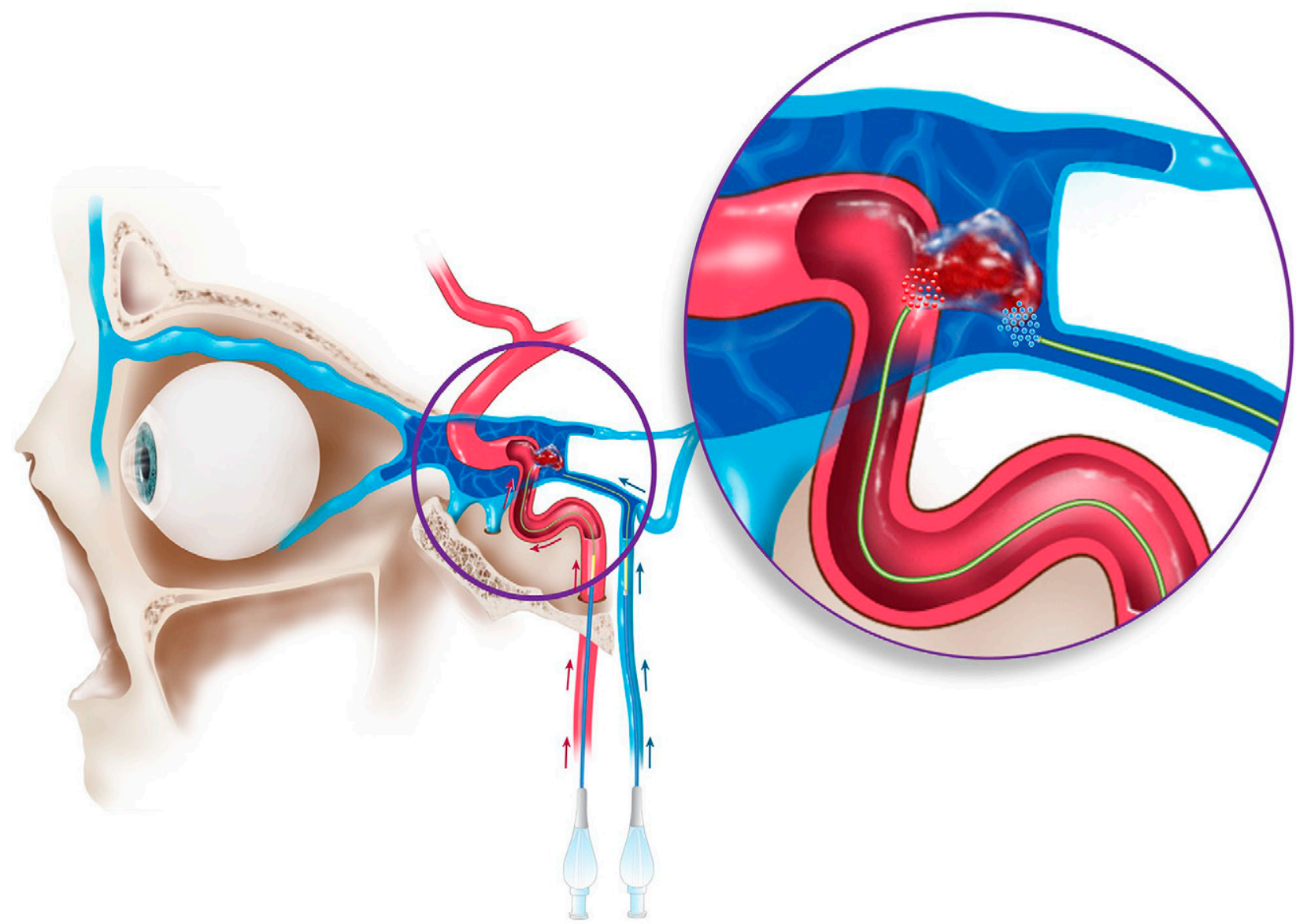

FIG. 2. An illustration depicting simultaneous transarterial and transvenous contrast injection technique with point of connection magnified inside the purple circle.

ipsilateral common carotid artery (CCA). With patent posterior communicating (PCom) artery, flow from basilar artery would opacify circle of Willis and from there to ICA and $\mathrm{CS}^{5}{ }^{5}$ However, for this technique to be used, patency of PCom and efficacy of compression were required.

Mehringer et al. ${ }^{6}$ used injection into the ipsilateral ICA while manually compressing the CCA proximal to the catheter tip to prevent retrograde flow of contrast into the CCA. Contrast injection and filming were performed simultaneously. Film rate that was used was two films per second for 3 seconds that helped to diminish the flow in the fistula temporarily and rendered the connection point. ${ }^{6}$

In 2012, Kurata et al. $^{7}$ used the route of inferior parieto-occipital vein in three cases to enter the CS where IPS was obstructed with an old, stiff thrombus. In 2013, Golitz et al. ${ }^{8}$ used algorithm of parametric color coding in which they applied color coding to two-dimensional angiographic images. Later comparison of regular angiographic images from initial, interventional and postinterventional stages with colorcoded images of the same fistula architecture and directionality of venous drainage pattern were better identified. This technique would require multistage intervention and increase complexity of utilizing resources. $^{8}$

In 2015, Yamauchi et al. ${ }^{9}$ performed transvenous embolization in three cases with the use of intravascular ultrasound (IVUS). In all cases, the IPS was occluded and IVUS helped identify the orifice of closed IPS to further guide the entry. This technique required an IVUS machine and specialized ultrasound catheter. ${ }^{9}$

In patients with rapid shunt flow, it would be difficult to reveal connection point with occlusion of ICA alone. Tsuji et al. ${ }^{10}$ in 2016 additional performed three-dimensional angiography of ipsilateral vertebral artery with combined with manual aspiration from balloonguided catheter closing off ICA, thus allowing visualization of the connection point. Last, in $2018 \mathrm{Jia}$ et al. ${ }^{11}$ published a new technique known as the frontier-wire probing technique to overcome an obstruction of the IPS. In this technique, they used 0.035 -inch guidewire to probe the obliterated IJV and used the created roadmap to insert a microcatheter into the $\mathrm{CS} .^{11}$

For simplicity of understanding, all the techniques are summarized in chronological order and year of publication in Table 1.

\section{Observations}

Despite many techniques that have been tried and reported, it is always challenging to overcome technical difficulties arising from tortuous anatomy of the IPS during treatment of CCFs via the preferred transvenous route. In our technique, we set up a concurrent arterial and venous systems. Given the arterial system is a highpressure system and the usual direction of flow of contrast would 
TABLE 1. Summary of techniques to overcome challenges of revealing fistulous point for transvenous route (described by author group and year of publication)

\author{
Authors \& Year \\ Technique
}

\begin{tabular}{ll}
\hline Huber, $1975^{5}$ & Injection into ipsilateral vertebral artery with simultaneous digital compression of the common carotid artery \\
\hline Mehringer et al., $1982^{6}$ & Contrast injection and slow filming performed simultaneously with ipsilateral carotid artery compression \\
\hline Kurata et al., $2012^{7}$ & Use of inferior parieto-occpital vein to enter the CS \\
\hline Golitz et al., $2013^{8}$ & Parametric color coding allowing to assess hemodynamics of fistula \\
\hline Yamauchi et al., $2015^{9}$ & Use of IVUS to identify orifice of inferior petrosal sinus \\
\hline Tsuji et al., $2016^{10}$ & $\begin{array}{l}\text { Three-dimensional angiogram of ipsilateral vertebral artery with simultaneous manual aspiration from } \\
\text { balloon-guided catheter closing off ICA }\end{array}$ \\
\hline Jia et al., $2018^{11}$ & Frontier wire probing to overcome obstruction of IPS \\
\hline Present case & Simultaneous contrast injection into the ICA and IJV \\
\hline
\end{tabular}

be from the high-pressure ICA to the low-pressure CS, injecting a simultaneous contrast bolus from venous end would oppose the arterial contrast flow. As a result, the fistulous connection that was previously obscured became visible allowing roadmap imaging guiding navigation into the CS.

\section{Lessons}

Use of simultaneous transarterial/transvenous contrast injection is relatively simple compared to other reported techniques to reveal an obscure connection point. It also shortens the duration of endovascular tools in the bloodstream and thus, reduces potential complication rate. Further use of this technique on larger study samples is important to validate its general use.

\section{References}

1. Gemmete JJ, Ansari SA, Gandhi DM. Endovascular techniques for treatment of carotid-cavernous fistula. J Neuroophthalmol. 2009;29(1):62-71.

2. Gonzalez Castro LN, Colorado RA, Botelho AA, Freitag SK, Rabinov JD, Silverman SB. Carotid-cavernous fistula: a rare but treatable cause of rapidly progressive vision loss. Stroke. 2016; 47(8):e207-e209.

3. Ng PP, Higashida RT, Cullen S, Malek R, Halbach VV, Dowd CF. Endovascular strategies for carotid cavernous and intracerebral dural arteriovenous fistulas. Neurosurg Focus. 2003;15(4):ECP1.

4. Henderson AD, Miller NR. Carotid-cavernous fistula: current concepts in aetiology, investigation, and management. Eye (Lond). 2018;32(2):164-172.

5. Huber P. A technical contribution of the exact angiographic localization of carotid cavernous fistulas. Neuroradiology. 1976;10(5):239-241.

6. Mehringer CM, Hieshima GB, Grinnell VS, Tsai F, Pribram HF. Improved localization of carotid cavernous fistula during angiography. AJNR Am J Neuroradiol. 1982;3(1):82-84.

7. Kurata A, Suzuki S, Iwamoto K, et al. A new transvenous approach to the carotid-cavernous sinus via the inferior petrooccipital vein. $J$ Neurosurg. 2012;116(3):581-587.

8. Gölitz P, Struffert T, Lücking H, et al. Parametric color coding of digital subtraction angiography in the evaluation of carotid cavernous fistulas. Clin Neuroradiol. 2013;23(2):113-120.
9. Yamauchi S, Nishio A, Takahashi Y, et al. An innovative technique for detecting the caudal end of occluded inferior petrosal sinus in cavernous arteriovenous fistula using intravascular ultrasonography-technical note. Neuroradiology. 2015;57(8):799-804.

10. Tsuji K, Nakagawa N, Fukawa N, Nagatsuka K, Nakano N, Kato A. A novel technique for identifying the fistulous point in a direct carotid-cavernous fistula. J Clin Neurosci. 2016;25:152-155.

11. Jia ZY, Song YS, Sheen JJ, Kim JG, Lee DH, Suh DC. Cannulation of occluded inferior petrosal sinuses for the transvenous embolization of cavernous sinus dural arteriovenous fistulas: usefulness of a frontier-wire probing technique. AJNR Am J Neuroradiol. 2018; 39(12):2301-2306.

\section{Disclosures}

Dr. Fraser reported grants from University of Kentucky, grants from the American Heart Association, Penumbra, Medtronic, Stream Biomedical, Fawkes Biotechnology, and Cerelux, LLC outside the submitted work. No other disclosures were reported.

\section{Author Contributions}

Conception and design: all authors. Acquisition of data: all authors. Analysis and interpretation of data: all authors. Drafting the article: all authors. Critically revising the article: all authors. Reviewed submitted version of manuscript: all authors. Approved the final version of the manuscript on behalf of all authors: Rupareliya. Statistical analysis: Fraser. Administrative/technical/material support: Rupareliya, Fraser. Study supervision: Fraser. Constant mentoring and critical presubmission feedback: Fraser.

\section{Supplemental Information}

Previous Presentations

Portions of this work were presented as a poster presentation at the Society of Neurolnterventional Surgery (SNIS) 18th Annual Meeting, Colorado Springs, CO, July 2021, and at the Society of Vascular and Interventional Neurology (SVIN) 2021 Annual Meeting, Phoenix, AZ, November 2021.

\section{Correspondence}

Correspondence: Chintan Rupareliya: University of Kentucky, Lexington, KY. chintan.rupareliya@uky.edu. 